\title{
Stereotactic radiosurgery for meningiomas
}

\author{
Lawrence S. Chin, M.D., Nicholas J. Szerlip, M.D., and William F. Regine, M.D. \\ Departments of Neurosurgery and Radiation Oncology, University of Maryland School of Medicine, \\ Baltimore, Maryland
}

\begin{abstract}
Meningiomas are benign tumors attached to the dura that typically have a slow growth rate. After gliomas, they are the most common primary tumor of the brain. They are ideal radiobiological targets because single-fraction radiation has a high biologically effective dose. Furthermore, a highly conformal radiation plan can provide effective treatment to the tumor while sparing the surrounding brain. Meningioma control rates range from 90 to $95 \%$, and the risk of morbidity is low. Radiosurgery is an excellent treatment for asymptomatic, small- to moderate-sized meningiomas. It is also ideal for patients with incompletely resected meningiomas, recurrent meningiomas, and risk factors precluding conventional surgery.
\end{abstract}

KEY WORDS • meningioma • stereotactic radiosurgery

First described by Cushing 5 in 1922 , meningiomas are benign tumors that arise from arachnoid cap cells and commonly attach to the dural coverings of the brain and spinal cord. Except for relatively rare instances of malignancy, these tumors maintain a separate plane from the pia, thus resulting in displacement rather than infiltration of normal brain. ${ }^{12}$ Large meningiomas, however, can parasitize the pial blood supply and even superficially invade the pia. $^{9}$ Its most common locations are convexity, falx/ parasagittal, cavernous sinus, sphenoid wing, anterior skull base, petroclival, tentorial, and foramen magnum. Depending on its location, the blood is usually supplied by a branch of the external carotid artery with some pial supply as well. ${ }^{34}$ Strategically located meningiomas can invade or completely occlude a dural venous sinus.

Meningiomas are the second most common primary brain tumor, accounting for $27 \%$ of the total, with an incidence of four per $100,000 .{ }^{1}$ Unlike most brain tumors, there is a female predominance. Their most common clinical presentations include headache, seizures, and focal neurological deficits specific to the lesion's location. Given their indolent growth, neurological symptoms are typically insidious and progress slowly.

The classic appearance of a meningioma on MR imaging is an isointense mass on $\mathrm{T}_{1}$-weighted sequences and an isointense to hyperintense signal on $\mathrm{T}_{2}$-weighted sequen-

Abbreviations used in this paper: $\mathrm{BED}=$ biological equivalent dose; GKS = gamma knife surgery; ICA = internal carotid artery; LINAC = linear accelerator; $\mathrm{MR}=$ magnetic resonance; $\mathrm{SRS}=$ stereotactic radiosurgery. ces, with homogeneous contrast enhancement. Migration of meningioma cells from the tumor margin along the dura appears as a tail of enhancement that is characteristic but not pathognomonic for the diagnosis. Hyperostosis or frank invasion of adjacent bone structures, particularly in the sphenoid wing, is also seen. The degree of edema can vary widely: small meningiomas are often associated with an inordinate amount of cerebral edema, whereas larger tumors may present with little to no associated edema. Causes of edema are likely multifactorial and due to brain invasion, venous congestion, and release of vasoactive peptides. ${ }^{24}$ Cerebral angiography can demonstrate arterial supply to the tumor and can also be used to reduce vascularity by administration of small particle or glue embolization. ${ }^{18}$ Angiography is also useful for determining if a venous sinus is patent, although MR imaging may provide similar information..$^{20}$

\section{SURGICAL TREATMENT}

Excision of meningiomas continues to be the standard therapy. Because meningiomas are benign tumors frequently with a defined border, complete resection theoretically accomplishes a cure of the disease. Furthermore, resection can reduce focal neurological deficits and provide relief from mass effect. The Simpson grade was designed to correlate completeness of resection with risk of tumor recurrence (Table 1) ${ }^{29}$ Even complete resection with a margin of uninvolved dura is associated with tumor recurrence. ${ }^{2}$ In addition, complete excision carries potential risks that may be significant depending on the location 
TABLE 1

Summary of Simpson grades and definititons

\begin{tabular}{cc}
\hline \hline Grade & Definition of Corresponding Resection \\
\hline I & $\begin{array}{c}\text { macroscopically complete resection w/ excision of dural } \\
\text { attachment \& abnormal bone } \\
\text { macroscopically complete resection w/ coagulation of } \\
\text { dural attachment }\end{array}$ \\
III & $\begin{array}{c}\text { macroscopically complete resection w/o resection or } \\
\text { coagulation of its attachment }\end{array}$ \\
IV & $\begin{array}{c}\text { subtotal resection } \\
\text { simple decompression of the tumor }\end{array}$ \\
\hline
\end{tabular}

of the tumor. Although complete resection of cavernous sinus meningiomas has been proposed, the debilitating and disfiguring cranial neuropathies, as well as potential ischemic injury, make this option untenable. ${ }^{6,7,22}$ Foramen magnum and petroclival meningiomas are associated with risk of lower cranial nerve dysfunction and cerebrospinal fluid leakage; the tumors that invade or occlude the posterior sagittal sinus and torcula carry unacceptable risks of venous infarction. The development of modern radiosurgical techniques has provided an adjunct, or in some cases, an alternative therapy to the conventional surgery.

\section{STEREOTACTIC RADIOSURGERY}

Stereotactic radiosurgery is defined as focused-beam radiation delivered in one session to an intracranial target and involving the use of spatially defined localization and a rigid head fixation device. A modification of this technique involves fractionated, smaller-dose radiation delivered stereotactically and is called stereotactic radiotherapy. Typically, stereotactic radiotherapy involves relocatable, nonrigid skull fixation for its stereotactic localization. As will be discussed in the proceeding section, the theoretical benefit of fractionation over SRS for treatment of meningiomas is minimal.

Stereotactic radiosurgery is predominantly delivered using one of two types of devices: the gamma knife or modified LINAC. In GKS, 201, ${ }^{60}$ Co sources are focused on the target by interchangeable helmets with 4-, 8-, 14-, or $18-\mathrm{mm}$ collimators. The treatment is created by the additive effect of multiple isocenters of radiation that results in a high dose of radiation delivered in a highly conformal manner. Similarly, LINAC can produce the same effect by moving a single beam of radiation in arcs around a patient's head. The size of the beam can also be collimated, thus improving conformity of the plan.

In GKS, a Leksell G stereotactic coordinate frame is placed after administration of a local anesthetic agent. Given the spatial constraints of the Leksell frame and the gamma knife helmet, it is critical to center the tumor in the frame; for parasagittal tumors, the base of the frame will need to be at the level of the orbits. A contrast-enhanced MR imaging study is then performed, or computerized tomography scanning in patients in whom MR imaging is contraindicated due to the existence of a device such as a cardiac pacemaker. Treatment planning is performed using the interactive computer program that also provides important information such as isodose curves, as well as tumor and prescription volumes with dose-volume histograms (Fig. 1). Following the treatment, the patient is observed overnight for immediate side effects such as seizures. We do not routinely treat patients with corticosteroid agents after radiosurgery, unless they are already receiving this medication. Follow-up examination involves MR imaging studies obtained at 3 and 9 months after treatment and then annually thereafter. Because of their indolent nature, meningioma regression is not expected in the first few years of observation.

\section{RADIOBIOLOGY AND DOSIMETRY}

A critical concept in understanding the radiobiology of SRS is the $\alpha / \beta$ ratio. Each type of tissue has a measurable $\alpha / \beta$ ratio that reflects the survival of cells in relation to the dose of radiation. This $\alpha / \beta$ ratio is the key to determining the BED, which allows different radiation fractionation schemes to be compared. The following formula is used: $\mathrm{BED}=\mathrm{nd}(1+\mathrm{d} / \alpha / \beta)$, where $n$ equals the number of fractions and $d$ equals the dose per fraction.

Meningiomas have a low $\alpha / \beta$ that is characteristic of late-responding tissues such as benign tumors and normal brain. A low $\alpha / \beta$ translates into a higher BED for any given single fraction of radiation. ${ }^{15}$ This means that benign tumors such as meningiomas can receive a lower dose of radiation, yet have the same biological effect as a higher single fraction targeting a malignant tumor. Fractionation schemes are less important in meningiomas because both the brain and tumor have similar late-responding properties, and therefore there is no biological difference in $\alpha / \beta$ to exploit. Additionally, the highly conformal nature of radiosurgery in conjunction with the distinct tumor-brain border allows the high BED of a single dose to be restricted to the tumor.

The $\alpha / \beta$ of different tissue types determines the optimal dose range for each tumor type. Other important factors in

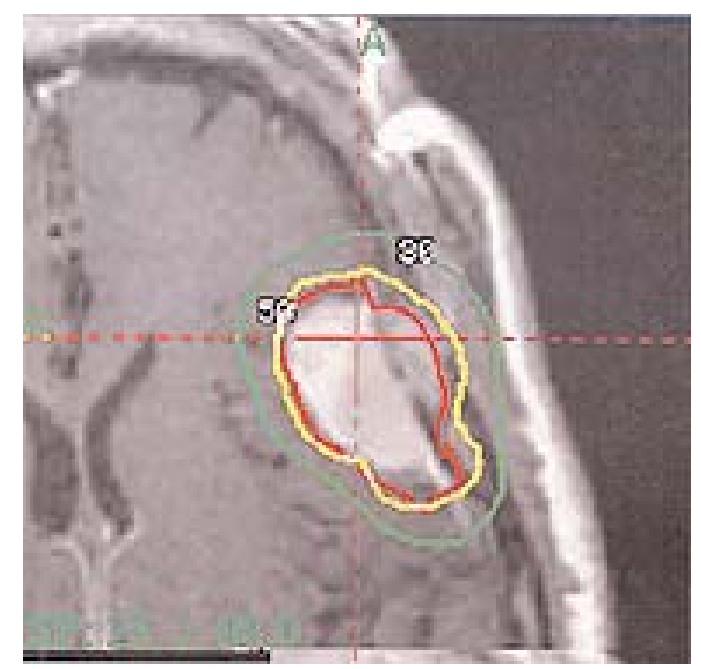

Fig. 1. Treatment plan for a sphenoid wing meningioma. The contrast-enhanced axial MR image demonstrates a sphenoid wing meningioma (red outline) with superimposed 30 and $50 \%$ isodose lines. The tumor has infiltrated into the overlying bone flap, which has been taken into account by the $50 \%$ isodose line (yellow line). 
determining the dose prescription include tumor volume, proximity to sensitive structures such as optic nerve, facial nerve, brainstem, and eloquent brain, and previous or future irradiation. The traditional optimal radiosurgery treatment size is less than $3 \mathrm{~cm}$ in diameter, but this is arbitrary. A larger treatment volume requires a lower dose to prevent undue risk of radiation necrosis. Treatment parameters that measure dose-volume relationships such as the $10-$ Gy volume are important to understand because they provide a benchmark for determining radiation risks. ${ }^{4} \mathrm{~A}$ suggested prescription dose at the tumor margin for meningiomas is $18 \mathrm{~Gy}(<1 \mathrm{~cm}), 16 \mathrm{~Gy}(1-3 \mathrm{~cm})$, and 12 to $14 \mathrm{~Gy}(>3 \mathrm{~cm})$. An alternative guideline is to follow the integrated logistic model for $3 \%$ risk of complication.

The accepted safe dose to the optic nerve is $8 \mathrm{~Gy}$, although doses up to 10 Gy have been reported without causing optic nerve injury. ${ }^{16,19,23,35} \mathrm{We}$ continue to recommend 8 Gy as the maximum optic nerve dose because of the serious consequences of radiation necrosis in this area. This constraint often lowers the margin dose for meningiomas in the parasellar/cavernous sinus region. The allowable dose to the brainstem and acoustic and facial nerves often restricts the dose given to meningiomas in the cerebellopontine angle and petroclival region. The brainstem can tolerate up to $15 \mathrm{~Gy}$, but facial nerve injury and decreased hearing can result with doses in this range when applied to acoustic tumors. ${ }^{8}$ Lowering the dose to $12 \mathrm{~Gy}$ in the treatment of acoustic neuromas has reduced the incidence of facial nerve injury to less than $5 \%{ }^{25}$ Depending on the volume, meningiomas along the skull base in the posterior fossa should receive a maximum of 12 to $16 \mathrm{~Gy}$.

In contrast to other cranial nerves, the motor cranial nerves in the cavernous sinus appear most resistant to radiation. Few oculomotor abnormalities are described after radiosurgical treatment involving the cavernous sinus. ${ }^{19}$ Trigeminal neuropathy is a more frequent complication, with a dose of 19 Gy appearing to be a threshold for injury in some series. ${ }^{19}$ In our currently recommended dose range, trigeminal nerve dysfunction should rarely be a problem.

Recently, critics of radiosurgery have pointed to anecdotal cases of malignant degeneration within a treated schwannoma or the development of a high-grade glioma in brain region adjacent to a treated meningioma as a harbinger of significant future risks. ${ }^{11,36}$ Although the risks of low-dose radiation for oncogenesis are well documented, at this time the failure of cases involving radiosurgery to rise above a few isolated reports makes the magnitude of this potential complication much lower than that known for conventional fractionated radiotherapy. ${ }^{27}$

\section{ANATOMICAL LOCATIONS}

Overall treatment results for meningiomas are excellent. Stafford, et al., ${ }^{32}$ reported on 190 consecutive patients with benign and malignant meningiomas treated by GKS between 1990 and 1998. Seventy-seven percent involved the skull base, and $12 \%$ were atypical or malignant. The median prescription isodose volume was $8.2 \mathrm{~cm}^{3}$ and the median tumor margin dose was 16 Gy (range 12-36 Gy). The median imaging and clinical follow-up periods were
40 and 47 months, respectively. The overall 5-year survival rates for patients with benign, atypical, and malignant meningiomas were 92,76 , and $0 \%$, respectively. The median survival for patients with malignant meningioma was 27 months. An overall local control rate of $91 \%$ was demonstrated with $56 \%$ of the lesions showing a decrease in size. The 5-year local control rates for benign, atypical, and malignant tumors were 93,68, and $0 \%$, respectively. Notably, tumor margin doses did not correlate with local control rates. The poor outcomes in cases of malignant meningiomas have been confirmed by others. Assuming that these tumors behave more like early-responding tissue types (with a high $\alpha / \beta$ ), radiobiology principles predict that higher doses will be needed to control malignant meningiomas compared with the benign form. Complications related to GKS were observed in $13 \%$ of patients, with new cranial neuropathies developing in $8 \%$ and radiation-induced $\mathrm{T}_{2}$-weighted MR imaging signal changes demonstrated in $3 \%$. These results are echoed in other large series of overall results with meningiomas. ${ }^{10,14}$

\section{Parasagittal Location}

Meningiomas attached to the convexity and falx along the posterior two thirds of the superior sagittal sinus are difficult to manage surgically because of the risk of sinus thrombosis and damage to adjacent draining veins. Radiosurgery is an attractive alternative to surgery as the primary treatment for small meningiomas as well as being useful as an adjunct for residual tumor after conventional resection. Kondziolka, et al., ${ }^{13}$ reported a 16-center retrospective study of 203 patients treated by GKS. The median tumor volume was $7.5 \mathrm{~cm}^{3}$, the median tumor margin dose was 15 Gy (range 9-32 Gy), and the median imaging follow-up period was 3.5 years. The 5-year local control rate was $67 \%$. In multivariate analysis the authors found that tumor volume greater than $7.5 \mathrm{~cm}^{3}$ and the presence of a neurological deficit predicted worse tumor control. The 3- and 5-year actuarial risk of developing symptomatic cerebral edema was $16 \%$, with the only significant factors being a previous neurological deficit and no history of resection. In every case, the edema resolved within a median interval of 15 months.

Singh, et al., ${ }^{30}$ have reported a higher incidence of symptomatic edema in cases of parasagittal tumors than in other tumor sites. Of 16 patients with adequate follow-up data, six (37.5\%) had perilesional edema and four (25\%) were symptomatic; no risk factors could be correlated with this finding. Our own anecdotal data support the notion that treatment of parasagittal and convexity meningiomas results in a higher incidence of perilesional edema. In addition to the aforementioned risk factors, treatment of parasagittal tumors exposes a larger volume of brain, including both the mesial and superior cortical surfaces, to radiation compared with skull base tumors.

Radiosurgery can be considered a sole treatment for tumors less than $3 \mathrm{~cm}$ that do not cause neurological deficits. Symptomatic tumors and those larger than $3 \mathrm{~cm}$ should be treated by resection and then by radiosurgery targeting areas of residual tumor. Additionally, a biopsy sample should be obtained or subtotal resection performed when the diagnosis is in question; dural metastases, sarcomas, and malignant meningiomas may mimic a benign 
meningioma. Tumors involving the superior sagittal sinus can be debulked, with the portion left in the sinus to be treated with radiosurgery. We have not noticed any complications related to treatment of tumor in any venous sinus. When designing the treatment plan, it is critical to determine the degree of infiltration along the falx both rostrocaudally and anteroposteriorly (Fig. 2). If feasible, the entire dural tail should be covered because the tumor tends to recur at the untreated margin. Although their greatest diameter is often small, meningiomas may be quite long, which will increase the tumor volume. It is strongly recommended that tumor outlines be drawn and volumes calculated before determining a final dose.

The best treatment for parasagittal meningiomas for which SRS is unsuccessful is not clear. In most cases, the pattern of failure will be at the margin of the original treatment volume or at a distant site, thus making additional radiosurgery an option. Inevitably, some overlap of brain irradiation will occur, raising the risk of radiation necrosis. When feasible, we recommend resection, with or without fractionated radiotherapy, of the recurrent tumor; we reserve repeated radiosurgery for unresectable tumor or the next tumor recurrence.

\section{Cavernous Sinus}

Although enthusiasm for radical cavernous sinus surgery peaked in the past decade, the high rates of morbidity resulting from the attempt to achieve a complete removal of tumor from the cavernous sinus, in addition to strides made in radiosurgical technique, have reversed this trend. In numerous clinical studies investigators have confirmed that radiosurgery is an effective and safe treatment for cavernous sinus meningiomas. ${ }^{3,17,21,26,28,31,32}$
Lee, et al., ${ }^{17}$ reviewed 176 consecutive patients treated with GKS (1987-2000). Eighty-three patients (52\%) underwent primary radiosurgery, and in $76(48 \%)$ at least one prior resection had been performed. The median tumor margin dose was 13 Gy (range 8-25 Gy). The actuarial 5- and 10-year tumor control rate was $93 \%$ for the entire group, whereas in the 83 patients in whom radiosurgery was the sole treatment the 5-year control rate was $96.9 \%$. Tumor volume decreased in $34 \%$ and was stable in $60 \%$. As noted previously, the oculomotor nerves are resistant to radiation-related effects; in fact, improved function was demonstrated in 25 patients. Complications occurred in 11 patients $(6.7 \%)$, with three patients experiencing delayed visual deterioration. In two cases, the recalculated optic nerve doses were $12 \mathrm{~Gy}$, which is higher than that normally considered safe. Leber, et al., ${ }^{17}$ analyzed cranial nerve toxicity in 50 patients who underwent GKS for various tumors. They reported an actuarial incidence of optic neuropathy of $0,26.7$, and $77.8 \%$ in patients who received less than $10 \mathrm{~Gy}, 10$ to $15 \mathrm{~Gy}$, and greater than $15 \mathrm{~Gy}$, respectively, to the optic apparatus. Ove, et al., ${ }^{23}$ found a $0 \%$ rate of optic nerve injury after treating their patients, who harbored parasellar lesions, with an optic nerve dose of less than 8 Gy.

Vascular injury in the cavernous sinus appears to be rare following SRS. Stafford, et al., ${ }^{32}$ reported permanent neurological deficits secondary to ICA injury in two of 66 patients with cavernous sinus meningiomas treated by GKS. In one patient 50\% stenosis of the ICA occurred 60 months after radiosurgery, and in the other patient a complete occlusion of the ICA developed 35 months after radiosurgery. The ICA radiation dose exceeded 25 Gy in both cases. Roche, et al., ${ }^{26}$ described one patient of 92 with a cavernous sinus meningioma in whom occlusion of

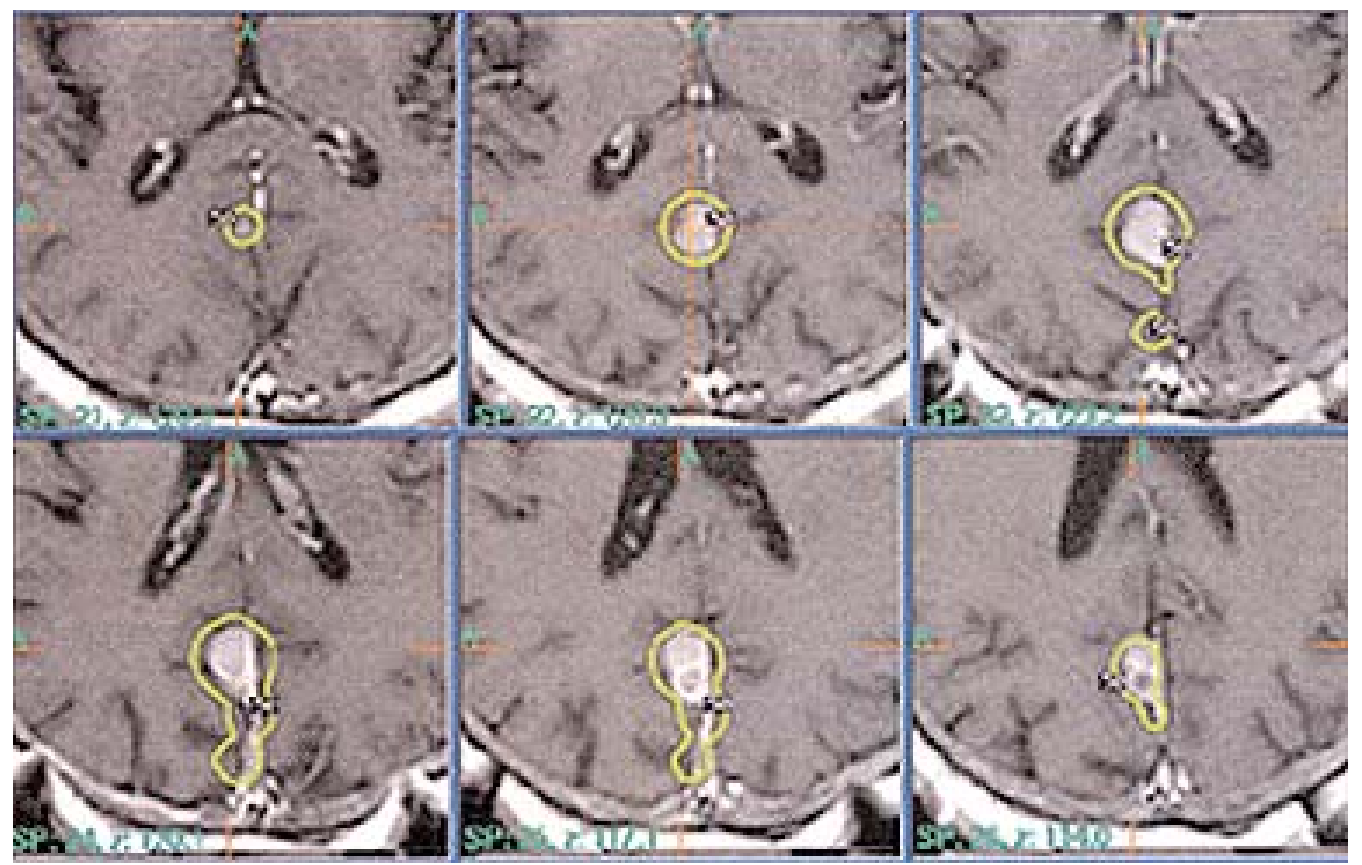

Fig. 2. Multiple panels showing the treatment plan for a falcine/parasagittal meningioma. The 50\% isodose line (yellow) covers the main tumor mass as well as its extension along the dura. The tumor did not involve the sagittal sinus. 
the ICA developed 13 months after GKS. She experienced a transient central facial palsy from which she recovered fully without treatment within a few days. The calculated ICA dose in her case was $36 \mathrm{~Gy}$. Our own results with cavernous sinus meningiomas have not been associated with any cases of carotid injury.

Linear accelerator-based radiosurgery has been shown to yield similar results in cavernous sinus meningioma. Spiegelmann, et al., ${ }^{31}$ reported on 42 patients who underwent SRS between 1993 and 2001 (a median follow-up time 36 months). The median tumor volume was $8.2 \mathrm{~cm}^{3}$, and the mean tumor margin dose was $14 \mathrm{~Gy}$. The 3- and 7 -year actuarial tumor control rate was $97.5 \%$. The rate of cranial nerve side effects was low: one patient $(2.4 \%)$ suffered a visual field cut at an optic pathway dose of less than $10 \mathrm{~Gy}$, and two patients (4.8\%) developed transient trigeminal neuropathies. An improvement in existing cranial nerve deficits was demonstrated in $20 \%$. Chang and Adler ${ }^{3}$ reported similar results in their series of 55 patients followed for 48 months after LINAC-based radiosurgery. A mean tumor volume of $7.33 \mathrm{~cm}^{3}$ was treated at a mean dose of $18.3 \mathrm{~Gy}$. The 2-year actuarial control rate was $98 \%$, and $5 \%$ of the patients developed new permanent symptoms. Neurological improvement was observed in $27 \%$.

Because of its high rate of tumor control and minimal rate of morbidity, SRS as the sole therapy should be considered the standard of care for cavernous sinus meningiomas smaller than $3 \mathrm{~cm}$ in diameter (Fig. 3). Craniotomy for debulking and decompressing the optic apparatus is still indicated for larger tumors and those that compromise vision. Additionally, tumors that abut the optic pathways, but do not cause compression or visual loss, may require debulking to achieve adequate separation from the optic nerves to allow an appropriate radiosurgical dose while the limiting radiation to the optic apparatus dose to less than 8 to $10 \mathrm{~Gy}$. It is important to visualize the entire optic pathway from the orbit to the optic chiasm and tract; in cases involving anterior cavernous sinus and medial sphenoid wing meningiomas, the dose limiting isodose line may contact the optic nerve in the orbit. Repeated radiosurgery may be necessary in patients in whom initial SRS fails because resection is not beneficial. Alternatively, fractionated radiotherapy can be attempted.

\section{Petroclival Location}

Petroclival region meningiomas are challenging to treat surgically because they are often intimately associated with multiple lower cranial nerves and the brainstem and because their extensive dural attachments along the skull base make complete resection impossible. Subach, et al., ${ }^{33}$ retrospectively analyzed 62 cases treated with GKS, of which 39 patients $(63 \%)$ had undergone at least one prior resection. The mean tumor volume was $13.7 \mathrm{~cm}^{3}$, and the mean tumor margin dose was 15 Gy. Neurological improvement was seen in $21 \%$, whereas $13 \%$ worsened. Tumor volumes decreased in $23 \%$, remained stable in $68 \%$, and increased in $8 \%$. New cranial nerve deficits developed within 24 months of GKS in five patients (8\%), but resolved completely in two.

Because petroclival meningiomas tend to present as larger tumors, surgical debulking plays a much greater role in their treatment than in cavernous sinus menin-
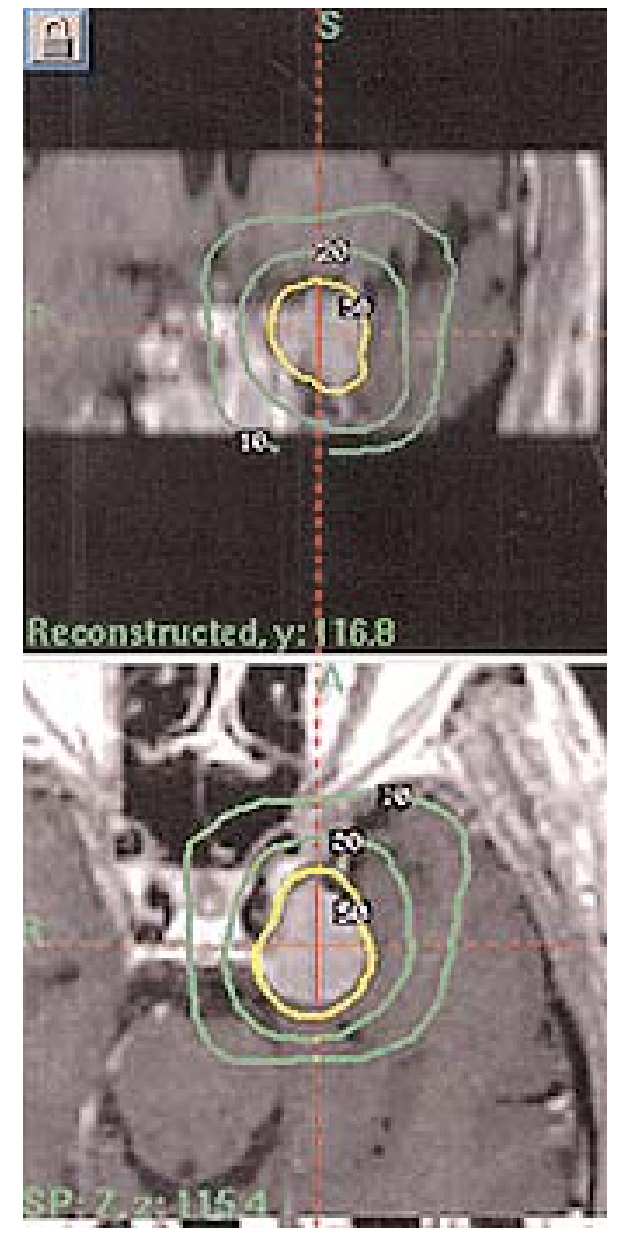

Fig. 3. Axial images with coronal reconstructions showing the treatment plan for a cavernous sinus meningioma. Upper: Image demonstrating the 10,20, and 50\% (yellow) isodose lines. The $20 \%$ line (green) is in contact with the optic chiasm and will limit the dose. Assuming that the $20 \%$ line receives $8 \mathrm{~Gy}$, the maximum dose to this tumor can be as high as $40 \mathrm{~Gy}$, or 20 Gy to the $50 \%$ line. In this patient, a 16-Gy margin dose was delivered to the $50 \%$, which means that 6.4 Gy was delivered to the edge of the optic chiasm. Lower: Image showing the tumor in relationship to the carotid artery, temporal lobe, and brainstem.

giomas. Small tumors, including those causing neurological deficit, may be appropriately treated with SRS alone because an improvement in symptoms can be seen. Large tumors should be surgically debulked first, and radiosurgery undertaken postoperatively for remnants. Location of the seventh to eighth cranial nerve complex should be identified so that the radiation dose in this area does not exceed 12 Gy.

\section{CONCLUSIONS}

The efficacy of SRS in the treatment of meningiomas is well established. Resection remains the primary treatment, however, for symptomatic and large tumors. A new treatment paradigm of conservative resection coupled with postoperative radiosurgery provides high tumor control rates and a low risk of complication, which ultimate- 
ly results in higher patient satisfaction. In current studies the authors have reported 5- to 10-year follow-up periods. Given the indolent nature of meningioma growth, the final validation of SRS awaits studies with 20-year follow-up data.

\section{References}

1. Central Brain Tumor Registry of the United States: Statistical Report: Primary Brain Tumors in the United States, 1995-1999. Chicago: CBRTUS, 2002

2. Chan RC, Thompson GB: Morbidity, mortality, and quality of life following surgery for intracranial meningiomas. A retrospective study in 257 cases. J Neurosurg 60:52-60, 1984

3. Chang SD, Adler JR Jr: Treatment of cranial base meningiomas with linear accelerator radiosurgery. Neurosurgery 41: 1019-1027, 1997

4. Chin LS, Ma L, DiBiase S: Radiation necrosis following gamma knife surgery: a case-controlled comparison of treatment parameters and long-term clinical follow-up. J Neurosurg 94: 899-904, 2001

5. Cushing $\mathrm{H}$ : The meningiomas (dural endotheliomas): their source, and favoured seats of origin. Brain 45:282-316, 1922

6. De Jesus O, Sekhar LN, Parikh HK, et al: Long-term follow-up of patients with meningiomas involving the cavernous sinus: recurrence, progression, and quality of life. Neurosurgery 39: 915-920, 1996

7. DeMonte F, Smith HK, Al-Mefty O: Outcome of aggressive removal of cavernous sinus meningiomas. J Neurosurg 81: 245-251, 1994

8. Foote RL, Coffey RJ, Swanson JW, et al: Stereotactic radiosurgery using the gamma knife for acoustic neuromas. Int J Radiat Oncol Biol Phys 32:1153-1160, 1995

9. Ginsberg LE, Moody DM: Meningiomas: imaging, in Wilkins RH, Reangachary SS, (eds): Neurosurgery, ed 2. New York: McGraw-Hill, 1996, Vol 1, pp 855-872

10. Hakim R, Alexander E III, Loeffler JS, et al: Results of linear accelerator-based radiosurgery for intracranial meningiomas. Neurosurgery 42:446-454, 1998

11. Hanabusa K, Morikawa A, Murata T, et al: Acoustic neuroma with malignant transformation. Case report. J Neurosurg 95: 518-521, 2001

12. Kleihues P, Burger PC, Scheithauer BW: Histological Typing of Tumours of the Central Nervous System, ed 2. Berlin: Springer-Verlag, 1993

13. Kondziolka D, Flickinger JC, Perez B: Judicious resection and/ or radiosurgery for parasagittal meningiomas: outcomes from a multicenter review. Gamma Knife Meningioma Study Group. Neurosurgery 43:405-414, 1998

14. Kondziolka D, Levy EI, Niranjan A, et al: Long-term outcomes after meningioma radiosurgery: physician and patient perspectives. J Neurosurg 91:44-50, 1999

15. Larson DA, Flickinger JC, Loeffler JS: The radiobiology of radiosurgery. Int J Radiat Oncol Biol Phys 25:557-561, 1993

16. Leber KA, Bergloff J, Pendl G: Dose-response tolerance of the visual pathways and cranial nerves of the cavernous sinus to stereotactic radiosurgery. J Neurosurg 88:43-50, 1998

17. Lee JYK, Niranjan A, McInerney J, et al: Stereotactic radiosurgery providing long-term tumor control of cavernous sinus meningiomas. J Neurosurg 97:65-72, 2002
18. Manelfe C, Lasjaunias P, Ruscalleda J: Preoperative embolization of intracranial meningiomas. AJNR 7:963-972, 1986

19. Morita A, Coffey RJ, Foote RL, et al: Risk of injury to cranial nerves after gamma knife radiosurgery for skull base meningiomas: experience in 88 patients. J Neurosurg 90:42-49, 1999

20. Nadel L, Braun IF, Muizelaar JP, et al: Tumoral thrombosis of cerebral venous sinuses: preoperative diagnosis using magnetic resonance phase imaging. Surg Neurol 35:189-195, 1991

21. Nicolato A, Foroni R, Alessandrini F, et al: Radiosurgical treatment of cavernous sinus meningiomas: experience with 122 treated patients. Neurosurgery 51:1153-1161, 2002

22. O'Sullivan MG, van Loveren HR, Tew JM Jr: The surgical resectability of meningiomas of the cavernous sinus. Neurosurgery 40:238-247, 1997

23. Ove R, Kelman S, Amin PP, et al: Preservation of visual fields after peri-sellar gamma-knife radiosurgery. Int J Cancer 90: 343-350, 2000

24. Paek SH, Kim CY, Kim YY, et al: Correlation of clinical and biological parameters with peritumoral edema in meningioma. J Neurooncol 60:235-245, 2002

25. Petit JH, Hudes RS, Chen TS, et al: Reduced dose radiosurgery for vestibular schwannomas. Neurosurgery 49:1299-1307, 2001

26. Roche PH, Regis J, Dufour H, et al: Gamma knife radiosurgery in the management of cavernous sinus meningiomas. J Neurosurg (Suppl 3) 93:68-73, 2000

27. Shapiro S, Mealey J Jr, Sartorius C: Radiation-induced intracranial malingnant gliomas. J Neurosurg 71:77-82, 1989

28. Shin M, Kurita H, Sasaki T, et al: Analysis of treatment outcome after stereotactic radiosurgery for cavernous sinus meningiomas. J Neurosurg 95:435-439, 2001

29. Simpson D: The recurrence of intracranial meningiomas after surgical treatment. J Neurol Neurosurg Psychiatry 20:22-39, 1957

30. Singh VP, Kansai S, Vaishya S, et al: Early complications following gamma knife radiosurgery for intracranial meningiomas. J Neurosurg (Suppl 3) 93:57-61, 2000

31. Spiegelmann R, Nissim O, Menhel J, et al: Linear accelerator radiosurgery for meningiomas in and around the cavernous sinus. Neurosurgery 51:1373-1380, 2002

32. Stafford SL, Pollock BE, Foote RL, et al: Meningioma radiosurgery: tumor control, outcomes, and complications among 190 consecutive patients. Neurosurgery 49:1029-1038, 2001

33. Subach BR, Lunsford LD, Kondziolka D, et al: Management of petroclival meningiomas by stereotactic radiosurgery. Neurosurgery 42:437-445, 1998

34. Taveras JM, Wood EH: Diagnostic Neuroradiology, et 2: Baltimore: Williams \& Wilkins, 1976, pp 159-189, 751-759

35. Tishler RB, Loeffler JS, Lunsford LD, et al: Tolerance of cranial nerves of the cavernous sinus to radiosurgery. Int J Radiat Oncol Biol Phys 27:215-221, 1993

36. Yu JS, Yong WH, Wilson D, et al: Glioblastoma induction after radiosurgery for meningioma. Lancet 356:1576-1577, 2000

Manuscript received March 21, 2003.

Accepted in final form April 14, 2003.

Address reprint requests to: Lawrence S. Chin, M.D., 22 South Greene Street, Suite S-12-D, Baltimore, Maryland 21201. email: lchin@smail.umaryland.edu. 